\title{
Dynamic Transmission Electron Microscope Study on the Crystallization of Ion-Bombarded Amorphous Germanium Thin Films
}

Tian T. Li ${ }^{1}$, Melissa K. Santala ${ }^{1}$, Leonardus Bimo Bayu Aji ${ }^{1}$, Sergei O. Kucheyev ${ }^{1}$, HuaiYu M. Cheng ${ }^{2}$ and Geoffrey H. Campbell ${ }^{1}$

1 Materials Science Division, Lawrence Livermore National Laboratory, Livermore, CA, USA

2 IBM/Macronix PCRAM Joint Project, IBM T. J. Watson Research Center, Yorktown Heights, NY, USA

The structure-properties relationships of amorphous group IV semiconductors such as germanium (a-Ge) have attracted wide research interests over the past decades. The materials have great technological significance thanks to a plethora of applications, and understandings of their structural properties provide key scientific insights for those with similar covalently bounded networks. Pulsed laser induced crystallization is a common method to evolve the nanostructure in a-Ge. In this study, we report the correlations between the differences in the nanostructure of a-Ge thin films created by ion bombardment, and the laser crystallization kinetics measured using dynamic transmission electron microscope (DTEM).

Previously, amorphous silicon (a-Si) prepared by different methods has have been shown to exhibit different medium range order (MRO), which gives rise to different mechanical properties and tendencies to structural relaxation under thermal annealing [1]. Changes in MRO can be measured using a statistical microscopy technique known as fluctuation electron microscopy (FEM), as shown in Figure 1. We sputter deposit $\sim 30 \mathrm{~nm}$ of a-Ge thin film, and subsequently alter the nanostructure by $\mathrm{Ar}^{+}$ion bombardment. We then observe the differences in MRO between the as-deposited and the treated samples using FEM.

Amorphous germanium is known to crystallize with rate as high as $\sim 10 \mathrm{~s}$ of $\mathrm{m} / \mathrm{s}$ during laser annealing. The movie-mode DTEM at LLNL provides us the unique capability to capture the structural evolution in situ during the crystallization process [2]. From a series of (up to 9) single-shot acquisitions in under $\sim 1 \mu \mathrm{s}$, we can track the movement of the amorphous-crystalline boundary (Figure 2) with temporal resolution unavailable in conventional TEM experiments. We collect the crystallization kinetics data of both asdeposited and $\mathrm{Ar}^{+}$-treated a-Ge samples, and map the differences to the changes in the MRO.

\section{References}

[1] B. Haberl et al, J. Appl. Phys. 110 (2011) 096104.

[2] G.H. Campbell, J.T. McKeown, and M.K. Santala, Appl. Phys. Rev. 1 (2014) 041101.

[3] This work was performed under the auspices of the U.S. Department of Energy, Office of Basic Energy Sciences, Division of Materials Sciences and Engineering for FWP SCW0974 by Lawrence Livermore National Laboratory under Contract DEAC52-07NA27344. 


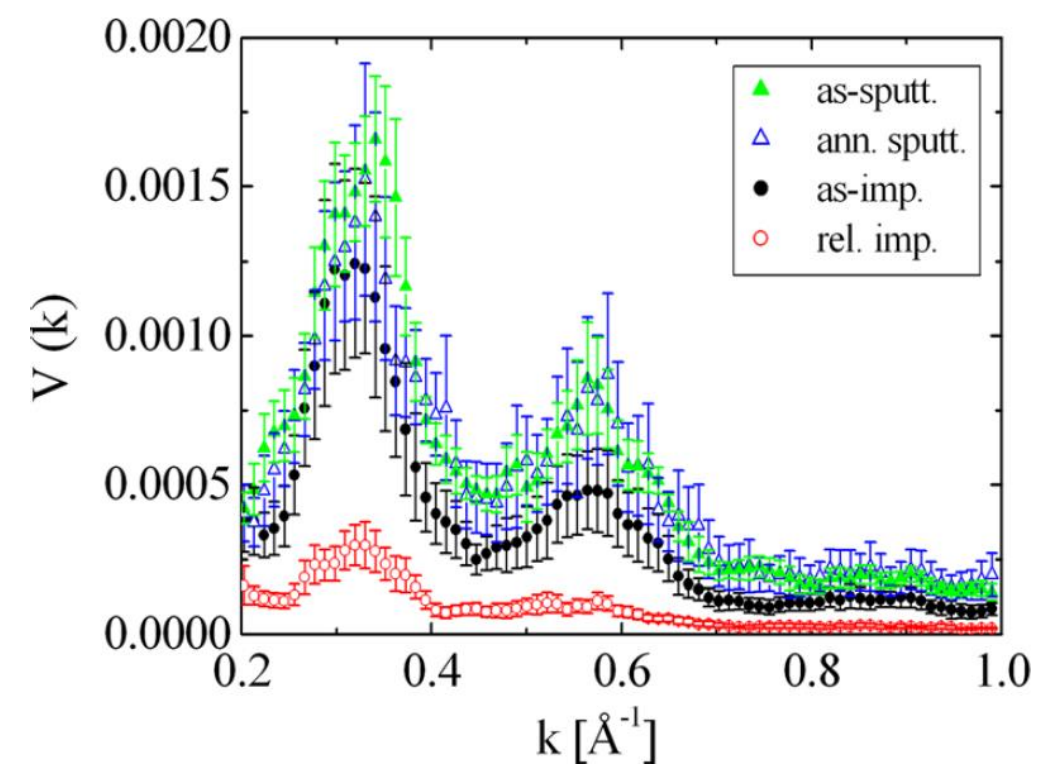

Figure 1: FEM variances $(\mathrm{V}(\mathrm{k})$ ) versus diffraction vector $(\mathrm{k})$ of four different amorphous Si samples: as-sputtered (as-sputt.), sputtered then annealed (ann. sputt.), as-implanted (as-imp.), and implanted then annealed/relaxed (rel. imp.). The differences in $\mathrm{V}(\mathrm{k})$ indicate the changes in the medium range order when the samples are subject to different preparation conditions and thermal treatments. Taken from [1], used with permission.
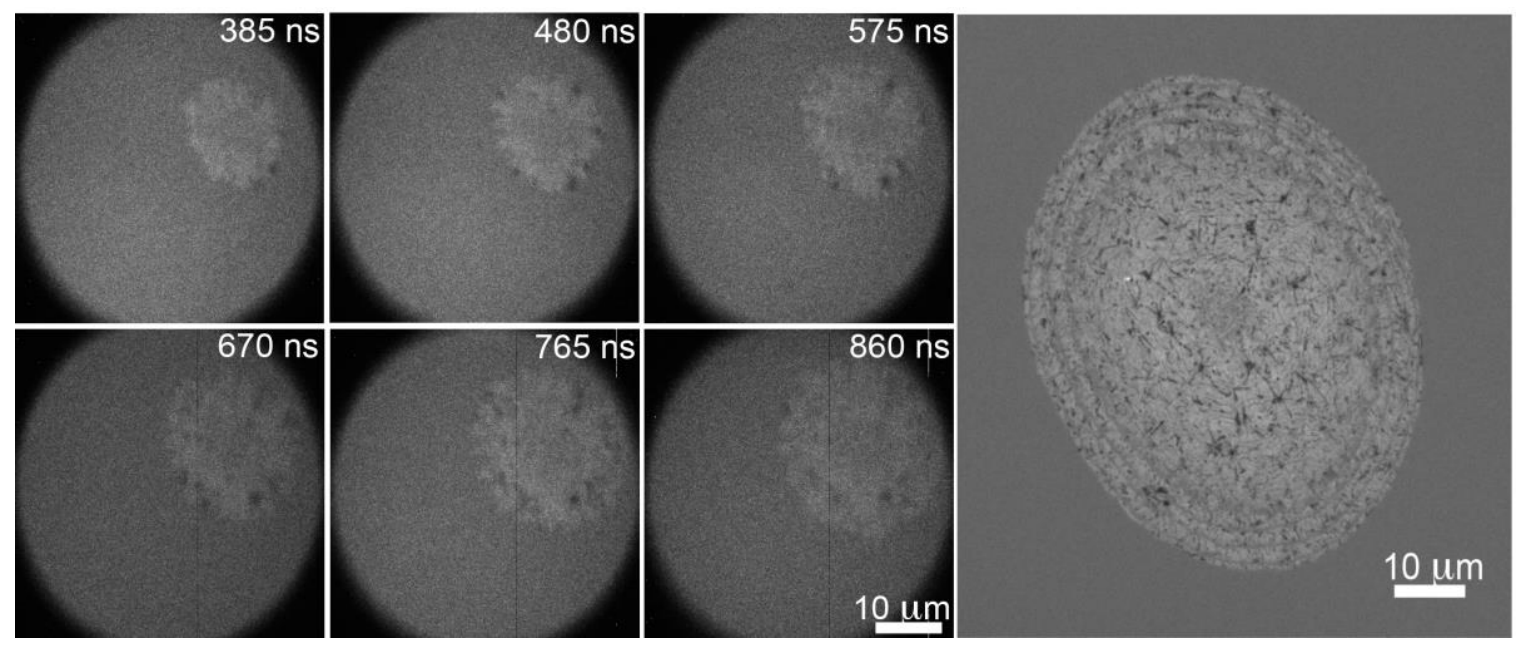

Figure 2: (Left) A series of images collected using Movie-mode DTEM during in situ laser crystallization of amorphous Ge. The images are collected with 20-ns exposure and $95 \mathrm{~ns}$ intra-frame time. The crystallization front moves at $\sim 20 \mathrm{~m} / \mathrm{s}$. (Right) conventional TEM image of the crystallized area after laser annealing. 